\title{
A NOTE ON A THEOREM OF B. H. NEUMANN AND S. YAMAMURO
}

\section{BILLIS}

If $\left\{G_{i} \mid i \in I\right\}$ is a class of groups and $D$ is a filter on the set $I$, then an equivalence relation can be induced on the Cartesian product $\prod_{i \in I} G_{i}$ by defining

$$
f \equiv g \quad \text { if and only if } \quad\{i \mid f(i)=g(i)\} \in D .
$$

It is not difficult to verify that the equivalence classes obtained from $\left.{ }^{*}\right)$ are the cosets of a normal subgroup $N_{D}$ of $\prod_{i \in I} G_{i}$, where $N_{D}=\{f \mid\{i \mid f(i)=e\} \in D\}$. The factor group obtained from $D$ in this manner is called a reduced product and is denoted by $\prod_{i \in I} G_{i} / D$. If $f \in \prod_{i \in I} G_{i}$ we define the support of $f$ by

$$
\sigma(f)=\{i \in I \mid f(i) \neq e\} .
$$

$G=\prod_{i \in I} G_{i}$ is said to have property $Q$ if whenever $N \triangleleft G$ and $f \in N$, then $\prod_{i \in \sigma(f)} G_{i} \subseteq N$.

B. H. Neumann and S. Yamamuro [2] showed that if $G$ is a finite non-Abelian simple group and $I$ is a set, then the Cartesian product $G^{I}$ has no countably infinite factor groups. By considering reduced products of groups we are able to give a shorter proof of a slightly more general result.

Lemma 1. If $G=\prod_{i \in I} G_{i}$ is a Cartesian product of groups, then every factor group of $G$ is a reduced product if and only if $G$ has property $Q$.

Proof. Suppose $G$ has property $Q$. Let $N \triangleleft G$. If $f \in G$, let $\phi(f)=\{i \in I \mid f(i)=e\}$. Then, letting $D_{N}=\{\phi(f) \mid f \in N\}$ it is not difficult to see that $D_{N}$ is a filter on $I$ and $G / N=G / D_{N}$.

Conversely, suppose every factor group of $G$ is a reduced product. If $N \triangleleft G$, then there exists a filter $D$ on $I$ such that $G / N=G / D$. If $f \in N$ and $h \in \prod_{i \in \sigma(f)} G_{i}$, then $\phi(f) \in D$ and $\phi(h) \supseteq \phi(f)$. Since $D$ is a filter, $\phi(h) \in D$. Hence, $h \in N$ and $G$ has property $Q$.

Lemma 2 is immediate from a result of Frayne, Morel and Scott [1, p. 210, Theorem 1.31]. This together with Lemma 1 gives us our desired result.

LEммa 2. If $\left\{G_{i} \mid i \in I\right\}$ is a class of finite groups and $D$ is a filter defined in $I$, then the reduced product $\prod_{i \in I} G_{i} / D$ is finite or uncountable.

Received by the editors December 2, 1968. 
Theorem. If $\left\{G_{i} \mid i \in I\right\}$ is a class of groups and $G=\prod_{i \in I} G_{i}$ has property $Q$, then $G$ has no countably infinite factor groups.

If $G$ is a finite non-Abelian simple group, then it is clear that $G^{I}$ has property $Q$. In fact, $\prod_{i \in I} G_{i}$ has property $Q$ if each $G_{i}$ is chosen from a finite set of non-Abelian simple groups. Hence, our result is slightly more general than that of B. H. Neumann and Yamamuro.

If $G=\prod_{i \in I} G_{i}$ has property $Q$ then each $G_{i}$ must be simple. However, the following example shows that if an infinite number of distinct non-Abelian simple groups is allowed, then $G$ no longer necessarily has property $Q$.

Example. Let $G_{i}=A_{2 i+3}, i=1,2, \cdots$. Then $G_{i}$ is simple and non-Abelian for all $i$. Let $f(i)=(123)$ for $i=1,2, \cdots$, and let $N$ be the normal closure of $f$ in $G=\prod_{i=1}^{\infty} G_{i}$. If $G$ has property $Q$, then $N=G$. However, if we define $g$ by $g(i)=(1 \cdot 2 \cdots 2 i+1)$, since conjugates of 3 -cycles are 3 -cycles, it is not difficult to see that $g \notin N$. Hence $N \neq G$ and $G$ does not have property $Q$.

Acknowledgements. The author wishes to thank Professor W.R. Scott for his help and encouragement and also Professor O. Kegel, through whom the author became aware of the existence of reduced products.

\section{REFERENCES}

1. T. Frayne, A. C. Morel and D. S. Scott, Reduced direct products, Fund. Math. 51 (1962/1963), 195-228.

2. B. H. Neumann and S. Yamamuro, Boolean powers of simple groups, J. Austral. Math. Soc. 5 (1965), 315-324.

Montana State University 